\title{
Long term visual outcome after arteriolar constriction in patients with branch retinal vein occlusion
}

\author{
Jiri Rehak ${ }^{\mathrm{a}}$, Ladislav Dusek ${ }^{\mathrm{b}}$, Martin Sin ${ }^{\mathrm{a}}$, Barbora Babkovaa , Zuzana Pracharova ${ }^{\mathrm{a}}$, Matus Rehak ${ }^{\mathrm{a}, \mathrm{c}}$
}

\begin{abstract}
Aim. To evaluate the final best corrected visual acuity (BCVA) in patients with branch retinal vein occlusion (BRVO) treated with either (1) laser arteriolar constriction (ACo) or (2) the standard treatment recommended by the Branch Vein Occlusion Study Group (grid laser photocoagulation (GLP) or only observation (if indication criteria for GLP were not met).

Methods. 358 BRVOs were treated in three different ways: early ACo $(n=133)$ performed $\leq 9$ weeks after onset of BRVO, late $\mathrm{ACo}(\mathrm{n}=62)$ performed $>9$ weeks after onset and controls which included GLP and observation $(n=163)$. The groups were further divided according to initial BCVA into: $\leq 0.1 ; 0.16-0.3$ and $\geq 0.4$.

Results. Based on the proportion of 1 year BCVA $\leq 0.1$ as a risk category, the results of early $\mathrm{ACo}(3.8 \%)$ were significantly better than for the controls $(22.7 \%, P<0.001)$ and late $\mathrm{ACo}(16.1 \%, P=0.006)$. The greatest difference in final BCVA was in patients with the worst initial VA $(\leq 0.1)$. In the intermediate initial BCVA group $0.16-0.3$, a significant difference was only found between the early $A C o$ group and controls $(P=0.007)$. There was no significant difference between any treatment group and prevalence of a final $B C V A \leq 0.1$ for patients with an initial $B C V A \geq 0.4$.

Conclusions. Early ACo significantly reduces the prevalence of a final BCVA $\leq 0.1$ in patients with BRVO and an initial $\mathrm{BCVA} \leq 0.3$ compared to controls (standard treatment treated with GLP or observed).
\end{abstract}

Key words: branch retinal vein occlusion, grid laser photocoagulation, arteriolar constriction, visual acuity, predictive factors

Received: October 3, 2012; Accepted: January 18, 2013; Available online: February 25, 2013

http://dx.doi.org/10.5507/bp.2013.008

${ }^{a}$ Department of Ophthalmology, University Hospital Olomouc and Faculty of Medicine and Dentistry, Palacky University Olomouc, Czech Republic

'Institute of Biostatistics and Analyses, Masaryk University, Brno

'Department of Ophthalmology, University of Leipzig, Leipzig, Germany

Corresponding author: Jiri Rehak, e-mail: rehakj@fnol.cz

\section{INTRODUCTION}

In general, branch retinal vein occlusion (BRVO) has a good prognosis; $50-60 \%$ of eyes are reported to have a final best corrected visual acuity (BCVA) $\geq 0.5$ even without treatment ${ }^{1-4}$. Currently, intravitreally applied steroids and VEGF inhibitors (Vascular Endothelial Growth Factor) are used in the treatment of macular edema (ME) secondary to BRVO as a treatment of first choice ${ }^{5-10}$. Grid laser photocoagulation (GLP) is also used if the ME persists or does not improve after intravitreal injections ${ }^{10}$.

Despite significant improvement in treating BRVO in recent years, there are still patients with chronic and/or recurrent ME leading to irreversible visual impairment. Several non-standard approaches have been described for the treatment of these patients ${ }^{1}$. One of these rarely used techniques is laser induced arteriolar constriction, also called the "crimping technique" ( $\mathrm{ACo}$ ).This procedure first described by L'Esperance in 1975 is based on the sacrificial constriction of the afferent arteriole in the occluded BRVO region ${ }^{11}$. Other authors have described their own modifications of ACo and have reported significantly improved BCVA in BRVO patients treated by this technique $^{3,12-15}$. The major limitations of these studies however, are small sample size and lack of control groups.
The aim of the present study was to evaluate the BCVA outcome in patients treated with ACo and to compare the results with the standard treatment following the Branch Vein Occlusion Study Group (BVO SG) recommendation ${ }^{16}$. A second question was whether early ACo in combination with GLP in the case of chronic ME, can reduce the prevalence of a final $\mathrm{BCVA} \leq 0.1$ compared with standard treatment regimen.

\section{MATERIALS AND METHODS}

We retrospectively reviewed the charts of 469 patients who were treated for ME due to BRVO between January 1988 and January 2010 at the Department of Ophthalmology, University Hospital in Olomouc. The study was approved by the Ethics Committee of the University Hospital in Olomouc and carried out according to the current Declaration of Helsinki.

Patients were included in the analysis if they had an (1) ME resulting from BRVO and (2) a minimum followup time of 12 months. Exclusion criteria were (1) previous laser or surgical treatment, (2) media opacities or any other ocular disease involving visual function and (3) incomplete charts in follow-up visits. Patients who had 
undergone any other treatment for ME (e.g., intravitreal steroids or anti-VEGF drugs) were also excluded from the statistical analysis. The diagnosis of BRVO was confirmed at the baseline visit (first examination in our department) by comprehensive ophthalmologic examination including slit-lamp biomicroscopy and fluorescein angiography (FA). In patients treated after June 2002, central retinal thickness was measured using OCT (OCT 2, and from 2006 OCT 3 Stratus, Zeiss Humphrey, USA).The results of the BCVA examination measured with a Snellen chart at the baseline visit and at 3,6 and 12 month follow-up visits were statistically evaluated.

Patients were divided into three subgroups according to different treatments: Group 1 "Early ACo" defined as ACo done within the first 9 weeks after onset of BRVO, group 2 "Late ACo" defined as ACo done after 9 weeks duration of BRVO and group 3 "Control group" including patients treated according to the BVO SG recommendation (patients with GLP if indication criteria defined as $\mathrm{BCVA} \leq 0.5$ due to ME persisting over 3 months were met and patients who were only observed in the case they did not meet these criteria). The groups of early and late ACo with $\mathrm{BCVA} \leq 0.5$ and a ME persisting over 3 months after baseline underwent additional GLP.

There was no randomization for treatment in the 3 subgroups. ACo is not a routinely used technique and the choice of treatment with ACo or standard approach was discussed with each patient. Following explanation of the ACo technique, the patient made the decision which treatment would be performed. The first ACo treatment was always done on the day of first examination at our department. The time period between first symptoms and the first treatment decided into which group (early or late $\mathrm{ACo}$ ) the patient would be analyzed.

All patients also received subsidiary medical treatment as $100 \mathrm{mg}$ pentoxyphyllin (3x1), $100 \mathrm{mg}$ salicylic acid $(2 \times 1)$, and $100 \mathrm{mg}$ ascorbic acid $(3 \times 1)$.The primary evaluated parameter was the final BCVA defined as BCVA 1 year after the baseline visit. The study also took into account, factors which could influence final BCVA such as age, sex, localization of occlusion (major temporal or macular BRVO), and the presence of retinal ischemia. Major BRVO was defined as BRVO of the temporal vein branch. If the clinical signs of occlusion were limited to the macular region and one or more macular venules were occluded, the patient was considered "Macular BRVO". Ischemic BRVO was defined according to the classification of the BVO SG as occlusion with a capillary nonperfusion larger than 5 disc diameters determined on FA ( ref. $^{17}$ ). In the case of large intraretinal hemorrhage preventing evaluation of the ischemic status, BRVO was classified as ischemic if cotton-wool spots were present.

\section{Laser arteriolar constriction technique}

We developed our own modification of ACo which consists in the application of coalescent coagulation spots through the afferent arteriole. The arteriole was coagulated at the site not obscured by the hemorrhage or not in the vicinity of the hemorrhage. Green argon laser (514.5 $\mathrm{nm}$ ) with the following parameters was used: spot size
200-500 $\mu \mathrm{m}$ (the beam was at least $50 \%$ larger than the vessel diameter itself) and exposure time 0.2-0.4 s. The intensity of the laser beam was set to a level that allowed access to a distinct narrowing of the arteriole. If the narrowing was insufficient, the power of the laser beem and/ or exposure time were increased and the vessel coagulated again. In reality, the narrowing was almost always only spasm of the vessel. Often no narrowing of the arteriole at the next visit could be seen even in the case of complete spasm during the primary ACo. (Fig. 1,2). The extent of coagulation of the arteriole was usually 1 disc diameter. The follow-up examinations were performed in the first 3 months after baseline every 2 weeks, later on up to 12 months after baseline every 1-2 months depending on the improvement in BCVA and ME. In the case of no evidence of treatment effect and insufficient narrowing of the arteriole, the ACo procedure was repeated at the same spot and/or at another arteriole or another part of the same arteriole. A treatment was considered effective if there was improved BCVA and/or reduced ME. The ACo procedure was repeated a mean of 3 times (1-6 times in early and 1-5 in late ACo resp.).

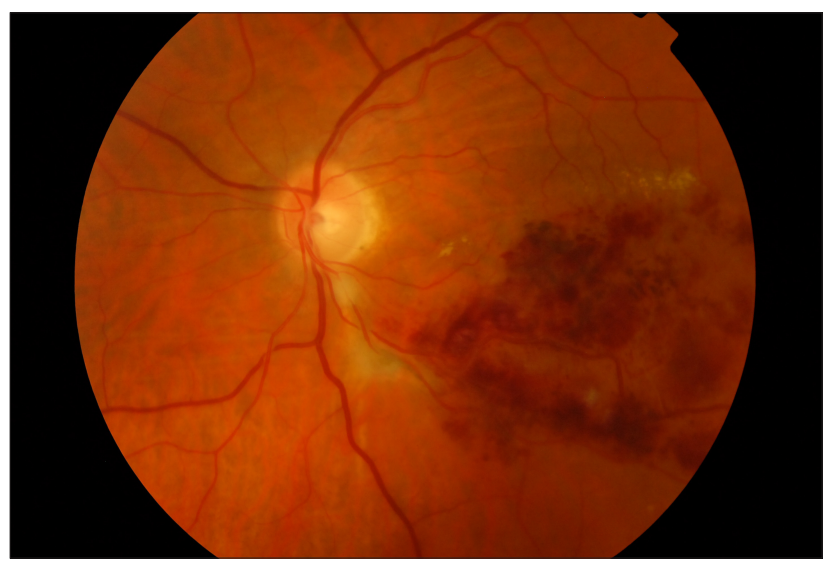

Fig. 1. Properly performed arteriolar constriction; total spasm of the arteriole can be seen. The image was taken immediately after photocoagulation.

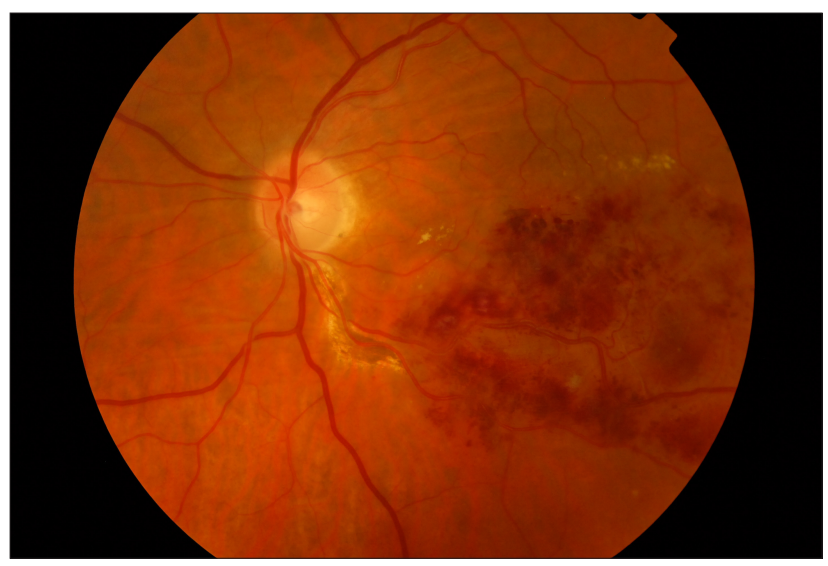

Fig. 2. Arteriole two weeks after arteriolar constriction. The image shows almost normal diameter of coagulated vessel with pigmented scar around the coagulated part of the arteriole. 


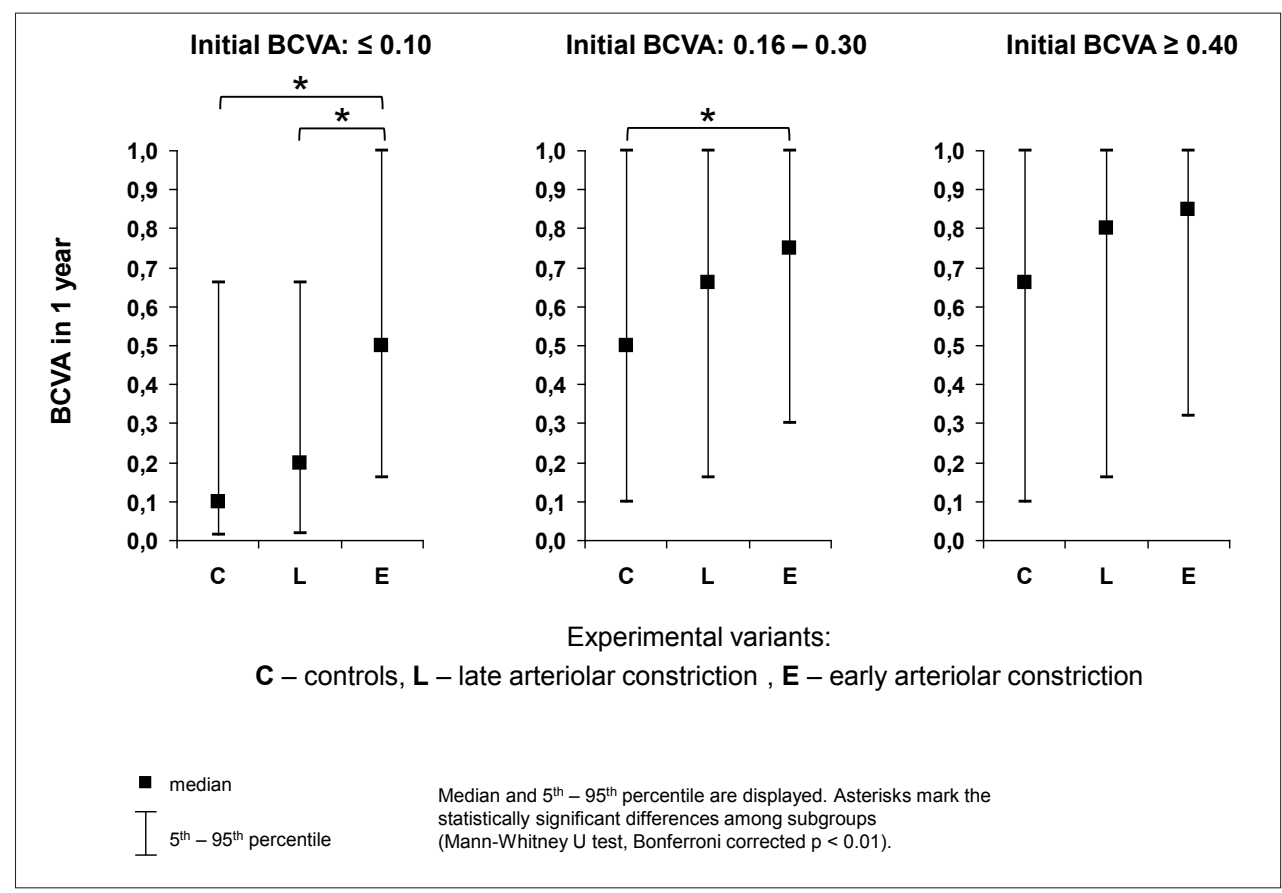

Fig. 3. Final best corrected visual acuity at 1 year follow up in the evaluated subgroups.

Table 1. Summary statistics of examined parameters.

\begin{tabular}{|c|c|c|c|c|c|c|c|c|c|}
\hline \multirow[b]{2}{*}{ Parameters $^{1}$} & \multirow{2}{*}{\multicolumn{2}{|c|}{$\begin{array}{c}\text { Grid photocoagulation } \\
\text { or observation } \\
\text { (control group) }[\mathrm{C}]\end{array}$}} & \multicolumn{4}{|c|}{ Arteriolar constriction } & \multicolumn{3}{|c|}{$P$ value $^{2}$} \\
\hline & & & & Late [L] & & $\operatorname{arly}[\mathrm{E}]$ & $\begin{array}{c}\text { C } \\
\text { vs. L }\end{array}$ & $\begin{array}{c}\mathrm{C} \\
\text { vs. E }\end{array}$ & $\begin{array}{c}\mathrm{L} \\
\text { vs. } \mathrm{E}\end{array}$ \\
\hline Sample size & & $\mathrm{N}=163$ & & $\mathrm{~N}=62$ & & $\mathrm{~J}=133$ & - & - & - \\
\hline Male sex & $48.5 \%$ & $(\mathrm{~N}=79)$ & $51.6 \%$ & $(\mathrm{~N}=32)$ & $48.9 \%$ & $(\mathrm{~N}=65)$ & 0.735 & 0.999 & 0.759 \\
\hline Age (years) & 62 & $(48$ & 62 & $(47 ; 74)$ & 58 & & 0.554 & 0.135 & 0.141 \\
\hline Age $>70$ years & $22.7 \%$ & $(\mathrm{~N}=37)$ & $14.5 \%$ & $(\mathrm{~N}=9)$ & $16.5 \%$ & $(\mathrm{~N}=22)$ & 0.199 & 0.241 & 0.835 \\
\hline Major BRVO & $88.9 \%$ & $(\mathrm{~N}=145)$ & $80.7 \%$ & $(\mathrm{~N}=50)$ & $81.2 \%$ & $(\mathrm{~N}=108)$ & 0.124 & 0.069 & 0.926 \\
\hline Ischemia & $33.7 \%$ & $(\mathrm{~N}=55)$ & $22.6 \%$ & $(\mathrm{~N}=14)$ & $27.1 \%$ & $(\mathrm{~N}=36)$ & 0.109 & 0.254 & 0.598 \\
\hline Initial VA & 0.300 & $(0.016 ; 0.700)$ & 0.250 & $(0.010 ; 0.700)$ & 0.100 & $(0.020 ; 0.500)$ & 0.136 & 0.020 & 0.172 \\
\hline Initial $\mathrm{VA} \leq 0.1$ & $27.6 \%$ & $(\mathrm{~N}=45)$ & $41.9 \%$ & $(\mathrm{~N}=26)$ & $51.9 \%$ & $(\mathrm{~N}=69)$ & 0.105 & 0.016 & 0.220 \\
\hline Final VA in 1 year & 0.660 & $(0.030 ; 1.000)$ & 0.500 & $(0.020 ; 1.000)$ & 0.900 & $(0.16 ; 1.000)$ & 0.325 & $0.007^{*}$ & $0.008^{*}$ \\
\hline VA $(1$ year $) \leq 0.1$ & $22.7 \%$ & $(\mathrm{~N}=37)$ & $16.1 \%$ & $(\mathrm{~N}=10)$ & $3.8 \%$ & $(\mathrm{~N}=5)$ & 0.359 & $<0.001^{*}$ & $0.006^{*}$ \\
\hline
\end{tabular}

${ }^{1}$ Categorical variables are summarized as $\mathrm{N}$ and percentage of a given category; continuous variables are described by median and by $5^{\text {th }}-95^{\text {th }}$ percentile range. ${ }^{2}$ Bonferroni corrected $P$ values $<0.01$ are typed bold and marked $\left({ }^{*}\right)$ as statistically significant.

\section{Statistics}

The non-parametric Kruskal-Wallis and MannWhitney U tests were used to evaluate the differences between groups in terms of BCVA and age. ML- $\chi^{2}$ test and Fisher exact test were used to test differences for binary or categorical variables. Time-related changes in BCVA were tested using the non-parametric Wilcoxon's matched pair test. The standard Bonferroni correction was applied on the outcome of all statistical tests with a final $P<0.01$ considered statistically significant. Logistic regression models were applied to quantify the association of examined prognostic factors with BCVA at 1 year. The multivariate regression was based on the stepwise selection algorithm, driven by the maximum likelihood ratio test. Effective cut-off values for continuous variables (age,
BCVA) were optimized on the basis of Receiver Operating Characteristics curves using categories BCVA at 1 year as risk $\operatorname{codes}^{18}$.

\section{RESULTS}

In total, the charts of 469 eyes of 469 patients were reviewed. 83 patients were excluded from the statistical analysis according to the exclusion criteria and a further 28 due to incomplete follow up examination. A total 358 eyes of 358 patients were analyzed. The baseline characteristics of the patients and subgroups are shown in Table 1. Based on the Bonferroni corrected boundary of statistical significance, $P<0.01$, there were no statistically 
Table 2. Percentage of a final BCVA $\leq 0.1$ in 1 year in experimental variants, stratified according to different initial BCVA.

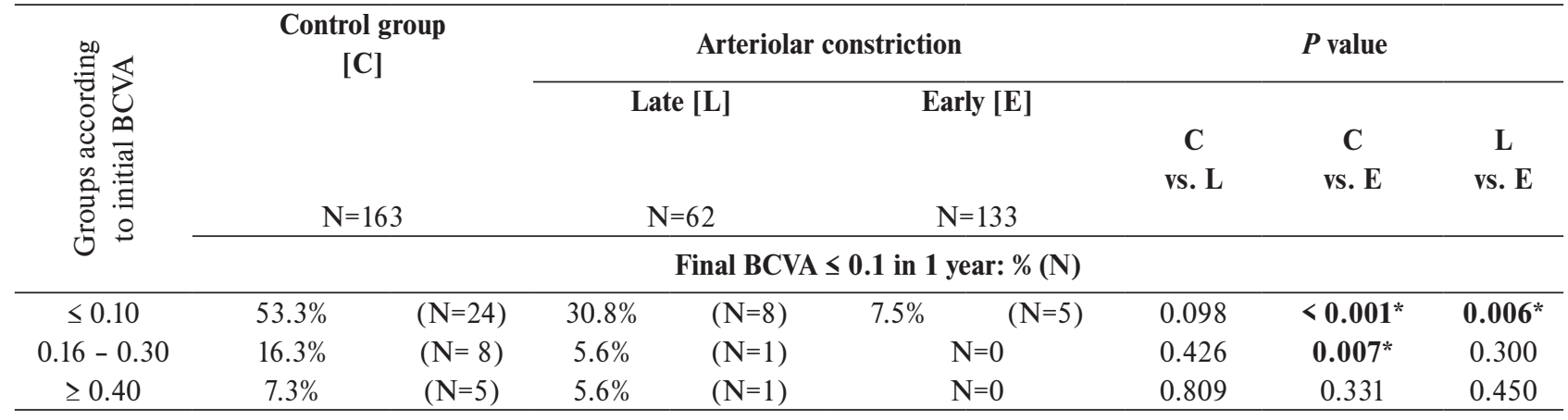

Statistical significance analyzed by Fisher exact test. Bonferroni corrected $P$ values $<0.01$ are in bold and marked $(*)$.

significant differences between the subgroups in baseline characteristics, including initial BCVA score. The groups could then be regarded as initially statistically comparable. In patients treated by $\mathrm{ACo}$, residual chronic $\mathrm{ME}$ requiring GLP was present in $46(35 \%)$ of early and 27 (44\%) of late ACo cases.

Comparison of initial and final BCVA revealed highly statistically significant increase in BCVA values during the 1 year follow-up in all three groups $(P<0.001$ for all comparisons). Table 1 shows the significant differences in final BCVA for individual subgroups. The final BCVA in the early ACo group was 0.9 which was significantly better than 0.66 for the control group. The final BCVA in the late ACo group reached 0.5 and did not significantly differ from the control group but was significantly lower than the early ACo group. Based on the proportion of patients with poor final BCVA defined as a BCVA $\leq 0.1$, the results of early ACo (3.8\%) were significantly better than the control group (22.7\%), and late ACo group (16.1\%). The differences remained highly significant after correction for multiple comparisons. The differences between control and late ACo in terms of outcome were not significantly different.

Comparison of treatment groups in terms of $\%$ of patients with a final BCVA $\leq 0.1$ at 1 year is summarized in Table 2. The comparison was performed within cohorts stratified according to initial BCVA, age, sex, type of occlusion and ischemia as potential predictors (only stratification for initial BCVA is shown in the table). Reflecting the results of our previous study, the three different initial BCVA subgroups $(\leq 0.1 ; 0.16-0.3$; and $\geq 0.4)$ were separately analyzed ${ }^{19}$. Compared to controls and late $\mathrm{ACo}$, the prevalence of final BCVA $\leq 0.1$ was statistically significantly lower in patients treated with early ACo (see Tab.1). No statistically significant difference between the late ACo group and the control group was found for any of the comparisons.

The results shown in Table 2, revealed that early ACo was most beneficial to patients with an initial BCVA $\leq$ 0.3 while there was no statistically significant improvement compared to controls in cohorts with an initial $\mathrm{BCVA} \geq 0.4$. The final BCVA outcomes compared with initial BCVA are also shown in Fig. 3. The greatest difference in final BCVA score was found for patients with the worst initial BCVA $(\leq 0.1)$. In this category, the early
ACo therapy had the greatest effect compared to both controls and late ACo. In patients with an initial BCVA 0.16-0.3, the quantitative differences between experimental groups were lower but the final effect of early ACo was still significantly better than for the control group. The effect of all treatments on the prevalence of final BCVA $\leq 0.1$ was not significantly different for patients with an initial $\mathrm{BCVA} \geq 0.4$.

Table 3 shows that several factors were significantly associated with the risk endpoint category coded as a final $\mathrm{BCVA} \leq 0.1$ in 1 year in the univariate analysis, even if corrected for multiple comparisons. A significant value of odds ratio (OR) was reached in the case of age (both as continuous variable and as category $>70$ years), male sex and initial BCVA category $\leq 0.1$. Early ACo therapy revealed highly significant OR as a positive factor associated with better therapeutic outcome. Late ACo therapy was not significantly distinguished from the control. Multivariate logistic regression was applied in order to recognize multivariate-adjusted significant factors which could have influenced the final therapeutic outcomes. Early ACo therapy, initial BCVA $\leq 0.1$ and age $>70$ years were found to be mutually independent factors significantly associated with final BCVA values. The positive effect of this treatment remains significant if evaluated in an age-adjusted model as well.

\section{DISCUSSION}

Even if anti-VEGF drugs and steroids are currently the first choice options in the treatment of ME secondary to BRVO (ref. ${ }^{5-10}$ ), recurrence of the ME and need for several re-injections are serious limitations. For this reason, the search for other options in the treatment of $\mathrm{ME}$ is an important clinical issue. One possible technique first described by L'Esperance is laser induced ACo (ref. ${ }^{11}$ ). In our chart review, we evaluated the outcomes in patients treated with ACo compared with the results of standard treatment following the recommendation of BVO SG.

In our previous study we showed the significant impact of initial BCVA on final visual result in patients with $\mathrm{ME}$ due to BRVO. We revealed that the final BCVA score significantly differed in the 3 groups of different initial BCVAs $\leq 0.1 ; 0.16-0.3$; and $\geq 0.4$ (ref. $^{19}$ ). For this rea- 
Table 3. Analysis of factors related to a final VA $\leq 0.1$ in 1 year as risk end-point.

\begin{tabular}{|c|c|c|c|c|c|}
\hline \multirow{2}{*}{ Parameter } & \multirow{2}{*}{ Reference category } & \multicolumn{2}{|c|}{ Crude odds ratio } & \multicolumn{2}{|c|}{ Multivariate-adjusted odds ratio $^{1}$} \\
\hline & & OR $(95 \% \mathrm{CI})$ & $P$ value & OR $(95 \% \mathrm{CI})$ & $P$ value \\
\hline Treatment & Controls & & & & \\
\hline Late ACo & (GLP or & $0.65(0.30 ; 1.42)$ & 0.281 & & \\
\hline Early ACo & observation) & $0.13(0.05 ; 0.35)$ & $<0.001$ & $0.08(0.03 ; 0.21)$ & $<0.001$ \\
\hline $\begin{array}{l}\text { Initial } \\
\mathrm{BCVA} \leq 0.10\end{array}$ & Initial VA > 0.10 & $4.86(2.54 ; 9.29)$ & $<0.001$ & $7.19(3.45 ; 15.02)$ & $<0.001$ \\
\hline $\begin{array}{l}\text { Age in years } \\
\text { (continuous) }\end{array}$ & - & $1.09(1.06 ; 1.13)$ & $<0.001$ & & \\
\hline Age $>70$ years & Age $\leq 70$ years & $6.29(3.33 ; 11.87)$ & $<0.001$ & $5.64(2.70 ; 11.76)$ & $<0.001$ \\
\hline Sex - male & Female & $0.41(0.22 ; 0.76)$ & 0.005 & & \\
\hline Major BRVO & Macular BRVO & $1.84(0.69 ; 4.86)$ & 0.219 & & \\
\hline Ischemia & No ischemia & $2.38(0.87 ; 6.45)$ & 0.235 & & \\
\hline
\end{tabular}

$\left(\mathrm{OR}=\right.$ odds ratio; $\mathrm{CI}=$ confidence interval). ${ }^{1}$ Only statistically significant ORs (Bonferroni corrected $\left.P<0.01\right)$ in multivariate model are displayed.

son, stratification according to initial BCVAs is necessary for the appropriate evaluation of treatment method. Reflecting these results, we analyzed the prevalence of final $\mathrm{BCVA} \leq 0.1$ in patients divided into three subgroups according to initial BCVA. The lowest prevalence of final $\mathrm{BCVA} \leq 0.1$ was found in patients with an initial VA $\geq$ 0.4 , which is in line with our previous study ${ }^{19}$. In this subgroup, no significant differences in prevalence of final $\mathrm{BCVA} \leq 0.1$ were found among the three different treatment approaches. In patients with an initial BCVA between 0.16-0.3, the prevalence of poor final BCVA was significantly lower only in the group of early ACo compared with controls. The highest prevalence of final BCVA $\leq 0.1$ was found in patients with an initial BCVA $\leq 0.1$. In this subgroup, the worst result was found in controls (53.3\%). Early ACo significantly reduced the prevalence of this poor final BCVA compared to both controls and late ACo. Further, from the multivariate analysis which took into account factors which could influence the final BCVA, we confirmed our previous finding that poor initial BCVA $(\leq 0.1)$ and age over 70 are strong negative independent predictors for a final BCVA $(\leq 0.1)\left(\right.$ ref. $\left.^{19}\right)$ while early ACo is a strong positive independent predictor.

Severe BRVO associated with large retinal hemorrhage is a therapeutic problem - GLP may lead to the development of hypercoagulation spots, epiretinal membrane and subsequent macular traction. Photocoagulation of the retina with extensive hemorrhage is questionable and contraindicated by some authors ${ }^{17,20-21}$. On the other hand, ACo can be done effectively in these cases, even outside the occluded retinal region under the condition that the coagulated arteriole supplies the occluded region.

In our experience, in the time interval up to 3-6 months, many eyes develop structural changes in the macula (disintegration of pigment epithelium layer, foveal cysts) which may lead to permanent decrease in BCVA. Here is probably the origin of the group of eyes with un- satisfactory final BCVA. This supposition is supported by our results in the treatment group with poor initial BCVA $(\leq 0.1)$ which showed statistically better visual results for early ACo compared to late ACo. These 2 groups differed only in the timing of the laser intervention (ACo was done in average 4 weeks in early ACo vs 4.2 months in late ACo after onset of BRVO).

Two independent studies have found that BRVOs with an initial BCVA of $\leq 0.25$ have a poor prognosis ${ }^{19,22}$. We consider such BRVOs to be prognostically unfavorable ${ }^{19}$. These BRVOs should be treated as soon as possible.

Even though the results of the ACo in our large patient group were positive, further prospective randomized studies are needed. A combination of ACo and anti-VEGF drugs in the treatment of ME in BRVO might be promising for achieving faster and more substantial reduction in the ME and fewer re-injections. This combination is the focus of our ongoing research.

\section{ACKNOWLEDGEMENT}

Authorship contributions: JR, MR: literature search; JR: manuscript writing; JR: study design; JR, MS, BB, ZP, MR: data collection; JR: data analysis and interpretation; LD: statistical analysis, figures; JR: final approval.

Conflict of interest statement: none declared.

\section{REFERENCES}

1. Rehak J, Rehak M. Branch retinal vein occlusion: pathogenesis, visual prognosis, and treatment modalities. Curr Eye Res 2008;33(2):111-31.

2. Gutman FA, Zegarra H, Zakov ZN. The natural course of temporal retinal vein occlusion. Trans Am Acad Opthalmol Otolaryngol 1974;78(2):178-92.

3. Jalkh AE, Avila PM, Zakka KA, Trempe CL, Schepens CL. Chronic macular edema in retinal branch vein occlusion: Role of laser photocoagulation. Annals Ophthalmol 1984;16(6):526-9. 
4. Morse PH. Prospective rationale for and results of argon laser treatment of patients with branch retinal vein occlusion. Annals Ophthalmol 1985;17(9):565-71.

5. Karagiannis DA, Karampelas MD, Soumplis VM, Amariotakis C, Georgalas I, Kandarakis A. Recurrence of macular edema in retinal vein occlusions after treatment with intravitreal ranibizumab (Lucentis). Can J Ophthalmol 2011;46(6):486-90.

6. Brown DM, Campochiaro PA, Bhisitkul RB, Ho AC, Gray S, Saroj N Adami AP, Rubio RG, Murahashi WY. Sustained benefits from ranibizumab for macular edema following branch retinal vein occlusion: 12-month outcomes of a phase III study. Ophthalmology 2011;118(8):1594-602

7. Haller JA, Bandello F, Belfort R Jr, Blumenkranz MS, Gillies M, Heier J Loewenstein A, Yoon YH, Jiao J, Li X-Y, Whitcup SM. Dexamethasone intravitreal implant in patients with macular edema related to branch or central retinal vein occlusion twelve-month study results. Ophthalmology 2011;118(12):2453-60.

8. Jaissle GB, Leitritz M, Gelisken F, Ziemssen F, Bartz-Schmidt KU, Szurman P. One-year results after intravitreal bevacizumab therapy for macular edema secondary to branch retinal vein occlusion. Graefes Arch Clin Exp Ophthalmol 2009;247(1):27-33.

9. The SCORE Study Research Group. A randomized trial comparing the efficacy and safety of intravitreal triamcinolone with standard care to treat vision loss associated with macular edema secondary to branch retinal vein occlusion: the standard care vs corticosteroid for retinal vein occlusion (SCORE) study report 6. Arch Ophthalmol 2009;27(9):1115-28.

10. Campochiaro PA, Heier JS, Feiner L, Gray S, Saroj N, Rundle AC, Murahashi WY, Rubio RG: Ranibizumab for macular edema following branch retinal vein occlusion: six-month primary end point results of a phase III study. Ophthalmology 2010;117(6):1102-12.

11. L'Esperance FA: Ocular photocoagulation. A stereoscopic atlas. St.Louis, CV Mosby Company, 1975, pp 215-22.
12. Rehak J, Vymazal M. Arteriolar constriction in the treatment of occlusion of the retinal branch of the vein.Our first experiences.(in Czech) Cesk Oftalmol. 1993;49(3):155-9.

13. Rehak J. Branch retinal vein occlusion: Early laser treatment. New approach. $\mathrm{XI}^{\text {th }}$.Congress of the European Society of Ophthalmology, Budapest, Hungary 1997;2:1157-60.

14. Rehak J: Branch retinal vein occlusion. Part 2. Early Treatment with Argon Laser. New Approach. New Trends Ophthalmol 1997;12:77-82.

15. Erdöl H, Akyol N. Arterial crimping in branch retinal vein occlusion with macular edema. Acta Ophthalmol Scand 2000;78(4):456-9.

16. Branch Vein Occlusion Study Group. Argon laser photocoagulation for macular edema in branch retinal vein occlusion. Am J Ophthalmol 1984;98(3):271-82.

17. Branch Vein Occlusion Study Group. Argon laser scatter photocoagulation for prevention of neovascularization and vitreous hemorrhage in branch vein occlusion. Arch Ophthalmol 1986;104(1):34-41.

18. Eng J. ROC analysis: web-based calculator for ROC curves. Baltimore, Johns Hopkins University [updated 2006 May 17; cited 2008 February 20]. Available from: http://www.jrocfit.org.

19. Rehak J, Dusek L, Chrapek O, Fric E, Rehak M. Initial visual acuity is an important prognostic factor in patients with branch retinal vein occlusion. Opthalmic Res 2011;45(4):204-9.

20. Finkelstein D. Argon laser photocoagulation for macular edema in branch retinal vein occlusion. Ophthalmology 1986;93(7):975-7.

21. Roseman RL, Olk RJ. Krypton red laser photocoagulation for branch retinal vein occlusion. Ophthalmology 1987;94(9):1120-5.

22. Jaissle GB, Szurman P, Feltgen N, Spitzer B, Pielen A, Rehak M, Spital $\mathrm{G}$, Heimann $\mathrm{H}$, Meyer $\mathrm{CH}$. Predictive factors for functional improvement after intravitreal bevacizumab for macular edema due to branch retinal vein occlusion. Graefes Arch Clin. Exp. Ophthalmol. 2011;249(2):183-92. 